\title{
Dimension availability with antecedent success or failure in concept identification'
}

\author{
Vladimir Pishkin \\ VA HOSPITAL AND UNIVERSITY OF OKLAHOMA SCHOOL OF MEDICINE
}

\begin{abstract}
Abstraet
Concept identification (CI) performance was evaluated under conditions where pattern dimensions were either available (DA) or not available (DNA), with three levels of complexity, and antecedent success or failure, i. e., misinformation feedback (MF) on a preceding task. CI was negatively influenced by DNA, antecedent MF, and irrelevant information. DNA, antecedent $\mathrm{MF}$, and Complexity significantly interacted, in pairs, to inhibit rate of information processing. Interpretation of results was that the three variables function as salient determinants of the potential universe of hypotheses which S may be considering in search of solution.
\end{abstract}

\section{Problem}

Quantitative theories of conceptidentification (CI) are predicated on S's sampling of information cues in a somewhat systematic manner. This sampling is dependent upon response tendency which is based on Ss current hypothesis as to which is the relevant dimension. According to Restle's (1961) model, S's hypotheses are tested, and if not confirmed, they are returned to the original set of hypotheses and are included for S'S reconsideration. Hunt's (1962) analysis of this process suggests that a hypothesis which is disconfirmed when $S$ errs, is immediately replaced by another one from S's set of hypotheses about solution. Thus, it may be assumed that even though a S may make an error based on a specific hypothesis, the probability of repeating such error does not change much, since there is no memory for past performance. In a recent experiment, Trabasso \& Bower (1964) gave support for "limited memory" assumption, demonstrating that Ss' average recall was less than one attribute out of six in each pattern of a simulated CI task. Nevertheless, there is evidence that availability of information from past trials facilitates CI solution (Bourne, Goldstein, \& Link, 1963) and that Ss gain less relevant information from availability of their past errors than from their correct responses (Pishkin \& Wolfgang, in press).

Since probability of correct response on any trial, according to Bourne \& Restle's model (1959), is derived from the proportion of cues conditioned to the correct set of cues, then it follows that the rate of learning should have inverse relationship to the number of hypotheses in the set. Presently, it is predicted that difficulty of CI problems will be increased when the number of hypotheses to be tested by $\mathrm{S}$ becomes greater as a result of unavailability of pertinent dimensions and failure on a preceding CI task.

This experiment was to assess CI performance under following conditions which were designed: (a) to vary the number of potential hypotheses $S$ has available for sampling, and (b) to test the influence of S's past success or failure in a CI task upon a subsequent CI performance.

\section{Method}

Ss were 72 male psychiatric nursing assistants with a mean age of 32.68 (range: $25-45$ ). No Ss were previously exposed to CI procedure. The procedure, general CI task, and apparatus have been described elsewhere in detail (Pishkin, 1960). Each S was required to categorize geometric patterns presented on the screen into two classes in accordance with relevant dimensions by depressing one of two response keys. For example, if color were relevant, solution would be to depress left key for all redpatterns and right key for all green ones, regardless of the other stimulus characteristics. When a dimension was neither relevant nor irrelevant, it appeared at only one of its levels. Irrelevant dimensions had a 0 correlation with correct response. The bileveled dimensions were as follows: form (square or triangle); size (large or small); number (one or two patterns); color (red or green); vertical position (top or bottom of the screen); horizontal position (left or right side of the screen); orientation (upright or tilted); and border (presence or absence of black, 1/8 in border on the pattern).

The task was self-spaced. As the pattern appeared on the screen, $S$ responded by depressing one of two unlabelled keys. Depression of the key automatically activated either left or right feedback light for 1 sec. directly above one of the keys to indicate correct response. The next pattern appeared 3 sec. following S's response.

The CI task consisted of two phases. In Phase I, Ss were exposed to 50 trials of CI task, either with or without misinformation feedback (MF), where patterns varied in only two dimensions, border and orientation. Phase I was subdivided into two conditions: (1) $0 \% \mathrm{MF}$ $(0-M F)$, i. e., a solvable problem where ss received "correct" feedback on all trials with respect to "orientation" as relevant dimension with border irrelevant, and (2) 50\% MF (50-MF). In 50-MF condition the feedback was programmed randomly with no relationship to either one of the two dimensions, so that the problem was unsolvable (Pishkin, 1960).

Upon completion of Phase I, S was then immediately told to start a "new" problem. Phase II was the CI problem proper with no MF, and Ss performed for 192 trials or until they made 16 consecutive correct responses. Complexity was varied by introducing 1,3 , or 5 irrelevant dimensions in combination with one of the relevant dimensions. Phase II was elaborated into two conditions, where all relevant and irrelevant dimensions were either available (DA), or not available (DNA) to S. In DA condition S was presented with eight slips of paper and told that one of the dimensions listed on the slips would help him solve the problem. All eight dimensions were presented regardless of problem complexity and were available for S's inspection throughout Phase II. In the DNA conditions, $S$ was told that he was to begin on a new problem and no other information was given. Three problems were utilized with three different, relevant dimensions, even though the Problems effect was not anticipated to be significant (Pishkin, 1960) and was introduced only to minimize spread of information among Ss.

This was a $3 \times 2 \times 3 \times 2$ factorial design, with three Problems, each with a different relevant dimension (form, size, or number), two MF conditions (0-MF or 50-MF in Phase I), three degrees of Complexity $(1,3$, or 5 irrelevant dimensions), and two conditions of Dimensions (DA or DNA). The Ss were assigned randomly to the 36 conditions.

\section{Results and Discussion}

Phase I was introduced merely to evoke S's differential expectations (success or failure) in Phase II. In 


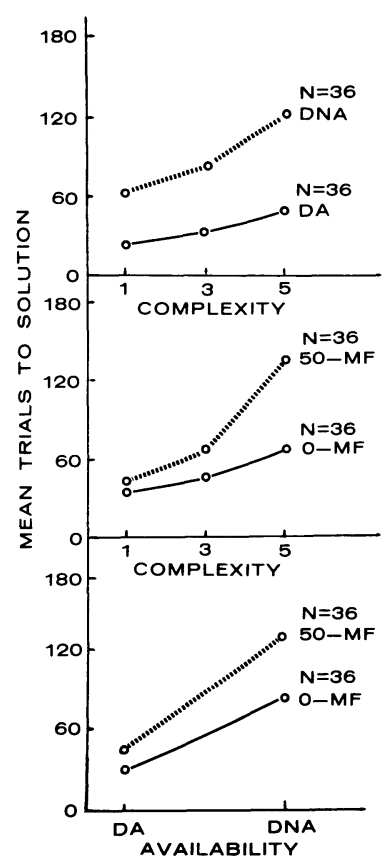

Fig. 1. Mean trials to solution as functions of dimension availability, MF and complexity interactions.

$0-\mathrm{MF}$ all Ss solved the problem by the 43rd trial, while in the 50-MF condition none of the Ss reached solution; and the asymptotic mean error value was 5.12 on the last block of 10 trials-closely approximating chance level and resulting in probability matching between random feedback and actual performance.

The dependent variable analyzed was number of errors in Phase II. Analysis of variance revealed that the main effects of MF, $F(1,36)=7.86 ; p<.01$, Complexity, $F(2,36)$ $=9.85 ; \mathrm{p}<.001$, and Dimensions, $\mathrm{F}(1,36)=5.81 ; \mathrm{p}<.05$, were all found to be significant. As expected, neither Problems effect nor any of its interactions were significant. Consistent with previous findings (Pishkin, 1960), orthogonal polynomial analysis indicated that only the linear component of Complexity was significant, $F(1,52)$ $=11.39 ; \mathrm{p}<.01$.

The discussion to follow will be concerned with the asymptotic values based on mean errors in the 12th block of 16 trials. The mean numbers of errors for conditions with 1,3 , and 5 irrelevant dimensions were $3.32,5.81$, and 8.74. The effect of MF was that Ss who were exposed to an unsolvable problem in Phase I (50-MF) produced more errors. Mean errors were: $0-M F=4.62,50-M F=7.29$. This finding suggests that 50-MF Ss approached Phase II problem with an expectation of greater universe of hypotheses than 0-MF group, after experiencing failure by not being able to validate any of their hypotheses in the first phase. An alternate explanation is that $50-\mathrm{MF}$ condition precipitated a decrement in S's motivation. A number of Ss in 50-MF indicated that they approached Phase II problem as though pattern dimensions had no relationship to feedback; e. g., some Ss looked for some systematic sequence of responses-leftkey, right key, etc. The Dimensions effect demonstrated a facilitative function, which may serve to limit the number of possible hypotheses Ss consider; mean errors were: $\mathrm{DA}=3.07, \mathrm{DNA}=8.65$. It appears that the $\mathrm{S}$ is able to focus more systematically in DA condition on one dimension after another until consistently reinforced, correct hypothesis emerges.

Interactions of MF $x$ Dimensions, $F(2,36)=7.82$; $\mathrm{p}<.01, \mathrm{MF} \times$ Complexity, $\mathrm{F}(2,36)=6.08 ; \mathrm{p}<.01$, and Dimensions $x$ Complexity, $F(2,36)=13.67 ; p<.001$, were all significant. The relationships between the interacting variables are demonstrated by the mean number of trials to solution within each condition as shown in Fig. 1. The MF $\times C \times D$ interaction was also significant, $F(2,36)=$ $5.08 ; p<.05$, revealing interdependence of the three main effects.

The DNA condition had a comparatively greater retarding effect on performance than DA as complexity increased (Fig. 1). Accordingly, unavailability of dimensions potentiated the function of irrelevant information since, from S's point of view, the number of dimensions was not limited. Likewise, 50-MF inhibited performance to a greater extent than $0-\mathrm{MF}$ as complexity increased.

In conclusion, it was demonstrated that CI performance is significantly influenced by $S$ 's success or failure : on a similar, antecedent task. Apparently, the number of hypotheses to be tested is increased when utilized dimensions are not available to S. Moreover, the importance of instructions as a mode of communicating information to S must not be overlooked. Presumably, the universe of cues to be "adopted" (Bourne \& Restle, 1959) markedly increases as a consequence of S's failure to solve a simple problem, especially when the varying dimensions are not communicated to Ss.

While a number of fundamental assumptions about CI behavior have been generated, it would seem important to consider S's set of hypotheses, in terms of his expectations; equally important is the degree to which dimensions are made available to Ss.

\section{References}

BOURNE, L. E., JR., GOLDSTEIN, S., \& LINK, W. E. Concept learning as a function of availability of previously presented information. J. exp. Psychol., 1964, 67, 439-448.

BOURNE, L. E., JR., \& RESTLE, F. Mathematical theory of concept identification. Psychol. Rev., 1959, 66, 278-296.

HUNT, E. B. Concept learning. New York: John Wiley \& Sons, 1962. PISHKIN, V. Effects of probability of misinformation and number of irrelevant dimensions upon concept identification.J. exp. Psychol., $1960,59,371-378$.

PISHKIN, V., \& WOLFGANG, A. Number and type of available instances in concept learning. J. exp. Psychol., in press.

RESTLE, F. Statistical methods for a theory of cue learning. Psychometrika, 1961, 26, 291-306.

TRABASSO, T., \& BOWER, G. Memory in concept identification. Psychon. Sci., 1964, 1, 133-134.

\section{Notes}

1. The author wishes to thank Marie Sherburne for her help in data collection. 